\title{
Cultivation of Modern Science-Technology-Engineering Concept for Engineering Students
}

\author{
Wenbo Zhu*, Jun Liu, Meijin Lin, Danfeng Chen, Shiya Liu, Aiyuan Li \\ Foshan University, Foshan, Guangdong, China \\ *Corresponding author. Email: zhuwenbo@fosu.edu.cn
}

\begin{abstract}
In order to enhance the sensitivity, consciousness and action ability in scientific research, technological innovation and engineering practice of engineering students, a four-year and consistent cooperative training program with science-technology-engineering concept is proposed. So as to enable engineering students truly understand the definition, connotation and relationship of the science, technology and engineering, to set up correct modern science-technology-engineering concept, and to improve their own scientific, technical and engineering literacy. Through this training program, engineering students are able to effectively master scientific, technical and engineering knowledge and specialties skills, to enhance their employability and entrepreneurial capabilities, and become professional and technical talents for economic and social development.
\end{abstract}

Keywords: Science-technology-engineering concept, Definition and connotation, Transformation path, Training Program, Engineering talent.

\section{工科大学生的现代“科学-技术-工程”观培养}

\author{
朱文博*, 刘军, 林梅金, 陈丹凤, 刘士亚, 李艾园
}

佛山科学技术学院, 佛山, 广东, 中国

*通讯作者. 屷箱: zhuwenbo@fosu.edu.cn

\section{中文摘要}

为了增强工科大学生科学研究、技术创新与工程实践的敏感性、自觉性与行动能力, 拟通过四年全程的、一贯 的“科学-技术-工程”观校企协同培养方案，使学生真正理解科学、技术、工程的定义、内涵以及相互关系，树 立正确的现代 “科学-技术-工程”观, 提高自身的科学素养、技术素养和工程素养, 切实掌握专业所需的科学、 技术、工程知识与技能, 提升就业和创业能力, 促使工科大学生成为对经济社会发展有用的专业技术人才。

关键词: 科学-技术工程观, 定义与内涵, 转化路径, 培养方案, 工程技术人才

\section{1. 引言}

随着新理论、新技术、新工艺、新材料、新产业、 新业态的不断涌现, 经济社会发展对 STE（科学、技 术、工程）知识与技能的需求程度日益提高。相关研 究表明, 现代社会中非 STE 领域的部分工作岗位 (如 营销和管理）对人才的科学、技术、工程相关知识储
备与技能需求不断增长, 至少需具备本科程度 STE 知识背景才能胜任实际岗位工作 ${ }^{[1]}$ 。对于工科大学生 而言, 他们未来的就业岗位绝大多数应该在 STE 工作 领域, 即使是在非 STE 工作领域就业中也有着一定的 优势, 而这就需要在大学教育教学中, 通过连贯、严 谨的科学教育教学, 使工科大学生通过学习科学、技 
术和工程的定义、内涵与相互联系，进而理解和树立 现代“科学-技术-工程”观的重要性, 促进科学、技术 和工程知识、推理和技能掌握和应用水平的提高。

欲促使工科大学生能够主动学习和掌握 STE 知识 与技能, 关键在于学生的现代“科学-技术-工程”观培 养。只有让工科大学生真正理解科学、技术、工程的 定义、内涵以及相互关系, 深刻把握科学、技术与工 程各自的特殊本质与特性, 才有助于工科大学生增强 科学研究、技术创新与工程实践的敏感性、自觉性与 行动能力。

\section{2. 现状分析}

早在 2013 年, 美国国家科学与技术委员会就向国 会提交了《联邦政府关于科学、技术、工程和数学 （STEM）教育战略规划（2013-2018 年）》[2][3]。但 历经多年的努力, 与其他工业发达国家相比, 虽然美 国在基础研究方面居于领先地位, 但是, 美国非科学、 技术、工程和数学领域工作人员的科学素养水平相对 较低, 他们普遍对科学不感兴趣, 严重影响了美国的 科学技术运用能力。然而, 德国、英国、日本等国家 把高质量的科学和数学教育看成是大学教育的重要 组成部分, 所培养的工程师和应用科学家的比例要比 美国大得多, 而且, 这些国家的中学、高等教育使大 多数学生的科学素养都达到了相当高的水平。

我国的工科教育和教学中也时常会忽视学生的科 学素养、技术素养与工程素养的培养, 为了片面提高 学生的专业技术水平, 广泛涉及专业及相关专业知 识, 把对专业知识的熟练掌握和应用作为衡量学生最 终学习结果的唯一标准, 使得学生越来越关注对专业 知识的机械应用, 而不去想为什么或者在工程实践中 提高科学知识水平。在他们看来, 科学理论、技术原 理与工程实践是相互分离的。在即将毕业或者已经毕 业的学生中最为流行的观点是: 大学所学的知识几乎 没什么用处, 在工程实际中什么都得从头开始学起。 实质上, 这样理解的毕业生在大学里真没学到什么, 因为他们并没有理解大学教育的真正含义与实质性 学习内容。

高等教育的目的不仅仅是向学生传授专业知识, 而更加重视科学思维和科学精神的培养, 树立正确的 “科学-技术-工程”观, 使学生在将来的工程实践中有 着科学的思维模式和思想方法。基础知识和专业知识 的学习只是这种科学教育的载体, 两者是一种依托关 系, 学生在学习基础知识和工程技术应用有关的专业 知识的同时, 逐渐培养科学的思维和严谨的科学精 神。因此, 尽管现代社会竞争压力大, 但要求应届大 学生有很高的专业知识和工程实践经验是不现实的, 而且仅仅把这一点作为判断是否接受过高等教育的 标准也是很片面的。

合格的大学毕业生在工程实际中应该是学习和接 受知识最快的人群, 也是进步最快的, 他们遇到问题
不是一味地生搬硬套以前的经验和技术, 而是认真地 思考, 把理论与经验有机地结合, 然后提出解决问题 的方法。在工程实践中，真正达到“不仅知其然，而 且知其所以然”的高度。这样, 才能在工程实践中不 断推进工程技术的进步和相关科学理论的发展。

一直以来, 普通高校的传统教育模式大多数是重 理论、重专业知识传授, 而轻科学思维、技术思维和 工程思维的培养教育, 尤其是在理论到实践的成果转 化方面缺乏足够的教育引导, 使得理工科学生只知公 式, 不知如何实现, 难以满足企业对合格工程技术人 才的需求。在工业 4.0、工业互联网和“中国制造 2025” 的战略背景下, 经济社会建设需要更多具有科学素养 的工程技术人才，非常有必要探索全新的知识学习、 能力导向、科研训练、应用创新的递进式培养, 产学 研深度结合、全程科研任务驱动的教育培养模式, 培 养卓越的工程技术人才。

\section{3. 现代“科学-技术-工程”观}

工科大学生只有切实理解和掌握科学、技术与工 程的定义、内涵, 以及三者之间的相互关系，才能树 立正确的现代“科学-技术-工程”观。

\section{1. 科学、技术与工程概念}

(1) 科学 (Science)。关于世界本质及其规律的 知识体系, 是人们对各种事实和现象进行观察、分类、 归纳、演绎、分析、推理、计算和实验，从而发现规 律, 并对各种定量规律予以验证和公式化的知识体系 [4]。

（2）技术（Technology）。关于利用和改造自然 的知识、经验、技巧和手段，是指人们利用现有事物 形成新事物, 或是改变现有事物功能、性能的方法。 技术应具备明确的使用范围和被其他人认知的形式 和载体, 如原材料（输入）、产成品（输出）、工艺、 工具、设备、设施、标准、规范、指标、计量方法等。

（3）工程（Engineering）。关于人工自然物设计 与建造实施的过程, 是一项精心计划和设计以实现一 个特定目标的单独进行或联合实施的工作, 人们要应 用科学知识和各种手段使自然力和自然物更好地为 人类服务。工程活动的内涵可以概括为“一个对象、 两种手段、三个阶段”。一个对象是指改造对象或建 造对象, 如建筑工程中的一栋大厦; 两种手段指技术 手段和管理手段，后者包括行政手段、经济手段和法 律手段等; 三个阶段包括: 策划阶段, 包括可行性论 证、规划、设计、调查、勘测等一系列前期工作; 实 施阶段，包括施工、制造、改造等；使用阶段，包括 使用、跟踪监测和维修保养等。

\section{2. 科学、技术与工程之间关系}

科学、技术与工程三者之间的关系如图 1 所示。 


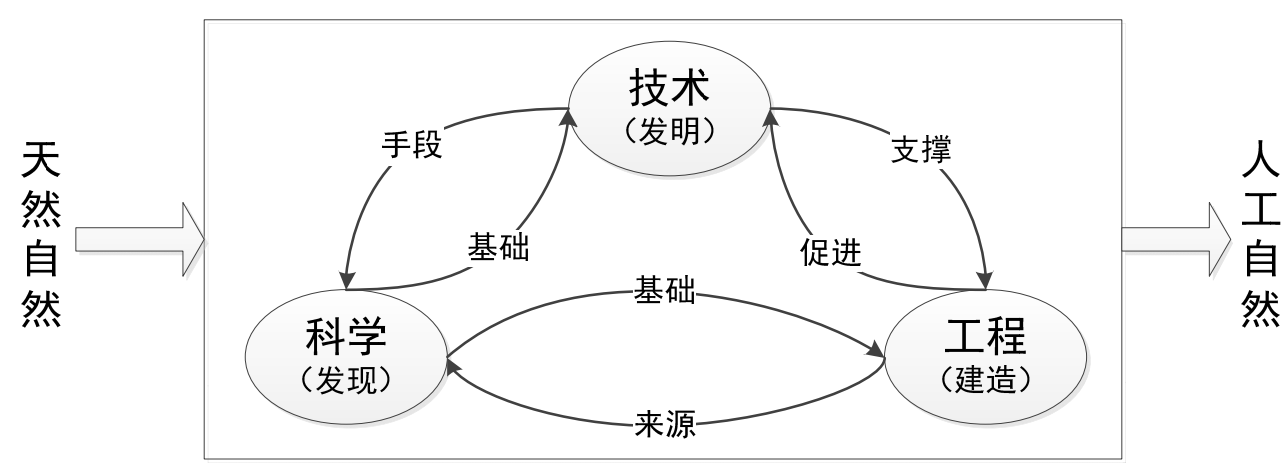

图 1 科学、技术与工程三者之间的关系

尽管科学、技术和工程都反映了人与自然之间的能动 关系, 但科学的本质是发现, 它使那些完全独立于人 存在的天然自然在实践中被人类所认识成为人化自 然; 技术的灵魂是发明, 它使一种崭新的人工自然的 诞生成为可能; 工程的核心是建造，使为人类服务的 人工自然物成为现实。因此, 科学、技术与工程三者 之间的关系可描述为: 科学是技术的基础, 技术是科 学研究的手段; 技术支撑工程的实施, 工程促进技术 的发展; 科学认识往往来源于工程实践, 而工程必须 建立在科学认识的基础之上。

实质上, 科学、技术和工程三者之间既有密切联 系, 又有明显区别, 可以从研究的目的与任务、研究 的过程与方法、成果性质与评价标准三个不同维度来 理解和掌握科学、技术与工程的定义与内涵, 以及三 者之间的区别, 理清从科学向技术的“转化”和从技术 向工程的“转化”路径, 使科学、技术与工程的研究成 果造福人类。

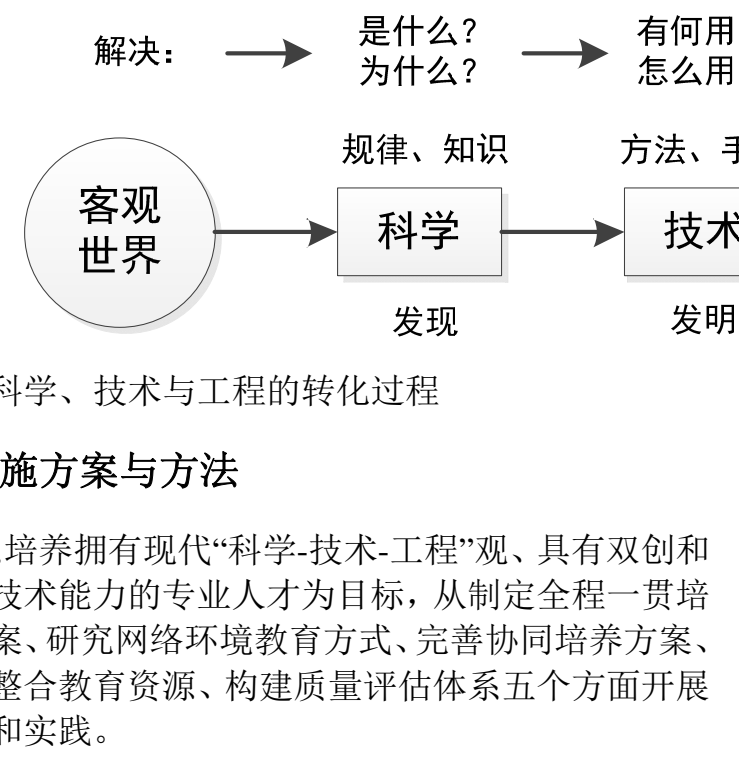

\section{1. 制定全程一贯培养方案}

制订四年全程、一贯的“科学-技术-工程”观培养方 案, 使学生养成科学、工程、技术整合学习的习惯, 树立正确的现代“科学-技术-工程”观。
人类从认识、发现客观世界（天然自然，包括人 类自身）的规律到适应、改造客观世界，创造出物质 文化成果 (人工自然) 有一连串的认识、转化、实施 过程，人类在实践过程中发现的客观规律称之为“科 学”。当人们运用这些规律去创造出成果时需要经过 两个转化过程: 第一个转化是应用规律去发明一些称 之为“技术”的方法和手段; 第二个转化是运用技术去 设计出所要求的工作目标并予以实施, 创造出物质文 化成果, 称为“工程”, 如图 2 所示 ${ }^{[5]}$ 。

对于工科大学生而言, 只有形成终生的“从科学向 技术转化”和“从技术向工程转化”, 以及“从技术发明、 工程实际抽取科学问题”的敏感性、自觉性和行动能 力, 才切实建立了现代“科学-技术-工程”观, 成为卓 越的工程技术人才。
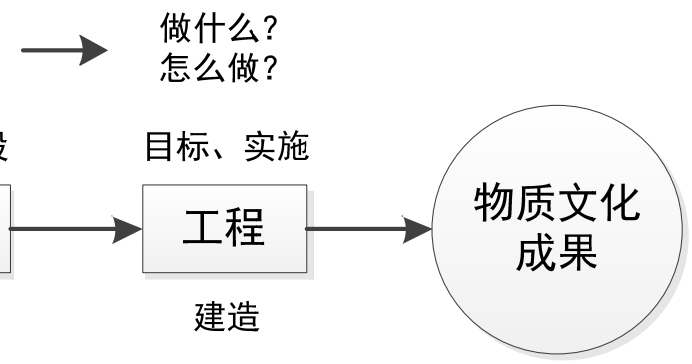

（1）从大学新生入学教育开始, 尤其在专业导论 课, 将科学、技术与工程的定义、内涵与相互关系传 授给学生, 形成初步的“科学-技术-工程”观。

（2）在大学四年专业教育过程中, 以基础课、专 业基础课、专业课为载体, 以科学知识为切入点、以 问题解决为出发点、以项目研究为立足点、以工程素 养为归宿点进行整合, 将科学、技术、工程知识与课 程知识讲解相融合, 使学生充分理解科学、技术、工 程联系与区别, 以及相互支撑与转化的基本原理。

(3) 在毕业设计阶段, 以实际项目驱动, 使学生 在“工程与技术”的实践中动手、动脑, 不断强化正确 的现代“科学-技术-工程”观。 


\section{2. 研究网络环境教育方式}

互联网及新一代信息技术可以用来模拟或仿真现 实中的科学、技术和工程 (即使是受限于学校的财力 和物力, 或者由于危险或者巨大耗损, 无法提供真实 的实践场景，也可以进行模拟或仿真），并充分考虑 它们对社会的影响, 从而避免未来的科学、技术和工 程发展到不利于人类的方向。网络环境教育方式方法 研究包括如下内容:

(1) 教学平台。在 2020 年初新冠肺炎防控期间, 腾讯课堂、腾讯会议、雨课堂、超星、MOOC 等一大 批网络平台纷纷出现在大、中、小学的在线教学实践 中, 不同平台有其自身的特点, 直播、互动、签到等 不一而足。需要深入对比分析, 䇻选出适合于工科大 学生教育的网络教学平台 ${ }^{[6]}$ 。尤其关注在 VR/AR、人 工智能等新一代信息技术应用方面有特色, 以便应用 于模拟现实中的科学、技术和工程。

（2）教学设计。根据网络教学特点, 结合课程的 教学大纲, 适当调整教学内容并制订网络教学授课计 划、教案、课后作业。教学设计更多采用情境式、模 拟式教学方式, 贴近学生的认知过程, 知识点突出, 时间适当控制, 增加互动环节、创新任务, 以学生为 中心，充分调动学生的积极性和创新思维。

（3）教学实施。教师精心设计教学内容, 制作课 件, 准备视频; 提前试播, 检测是否正常, 发布预习 作业和共享资料; 学生要明确每一次的学习目标, 提 前登陆教学平台做好上课准备; 课前 15 分钟, 教师 将课程 PPT 首页放在直播课屏幕上, 并播放学生喜欢 的音乐进行课堂预热。检查在线学生情况, 及时提醒 未到课的学生。讲解新知识之前, 可以进行简单的作 业点评和上堂课内容回顾。在新知识的讲解中, 教师 根据教学内容采用讲解、演示、讨论或视频播放等多 种教学方式。

(4) 教学测评。研究基于行为分析的网络教学监 督评估方法, 根据学生在线学习时电脑或智能终端的 状态、操作行为等信息数据对上课效果进行评估。网 络教学监督评估包括线上课程运行状态监测、学生行 为监测等 ${ }^{[7]}$ 。运行状态监测包括界面监测和音量监测。 行为监测主要是通过对输入设备监测实现, 包括滑 屏、鼠标监测、键盘监测、摄像头监测，等等。

\section{3. 完善协同培养方案}

现代工科大学生知识结构应具有如下特征: 能符 合现代科学技术发展特点; 能适应现代经济社会发展 趋势; 能体现现代教育思想要求; 能满足现代教育环 境需要。据此, 工科大学生应具有多维的知识结构 ${ }^{[8]}$, 如图 3 所示。

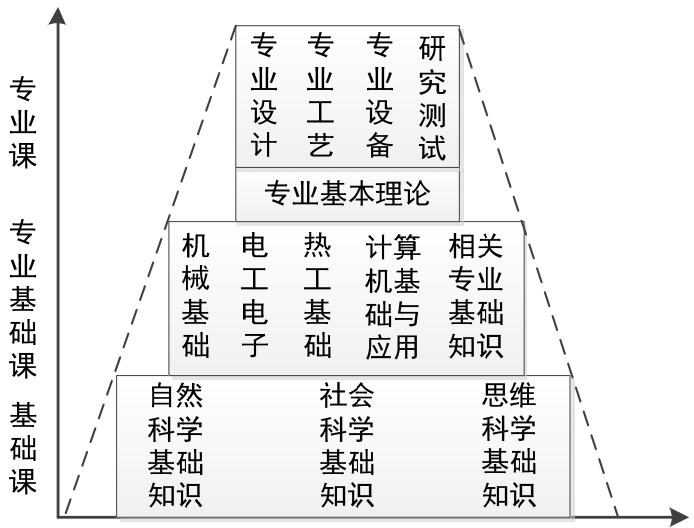

图 3 工科大学生知识结构模型

从图 3 的知识结构模型可以看出, 横向呈宽广型 结构, 基础课既要保证必要的系统性和一定的深度, 更要注重更新基础课教学内容, 使之能反映学科理论 的最新成就, 让学生获得自然科学规律与技术科学的 基本知识, 受到科学思维方法和运算、实验、设计、 数据处理等基本技能的训练。纵向知识呈梯形结构, 包括基础课、专业基础课、专业课, 专业课是体现专 业教学水平的主要标志, 为了扩大专业知识面, 提高 专业知识和技能水平。

在不断完善的协同培养方案中, 企业应深度参与 到整个工科技术人才的培养过程, 以年度为周期对人 才培养方案进行不断修订, 通过以下三个环节进行迭 代性修订和调整。

(1) 问卷调查。至少每年组织一次对工程技术岗 位人才的职业能力调查, 调查内容由学校和行业协会 共同组织设计, 由行业协会组织面向企业调查。收集 回来的问卷由学校组织汇总分析, 根据分析结果组织 专业教师对现行开出的课程内容进行检讨、论证, 确 定下一届学生开设课程从内容、组织形式到考核形式 的具体调整内容。

（2）需求调研。由行业协会组织面向相关企业进 行工程技术人才需求意向调研, 调研内容由学校和行 业协会共同研究制定，经行业协会收集汇总后形成统 计分析报告。

（3）专家论证。由学校和行业协会联合牵头, 从 企业中遴选一批行业专家，会同骨干教师一起对职业 能力、调整建议稿进行充分论证, 最终确定付诸实施 的方案。

通过问卷调查、需求调研、专家论证机制, 对所 开设课程内容进行及时有效调整。协同培养方案中工 艺性内容不宜过多过细过深, 但专业知识面可以更宽 一些。要把最新的专业技术成就及时地补充到教学内 容中, 使学生了解学科专业的前沿动态。注重专业课 教学的实践性, 结合校企合作, 在生产实习、专业课 程设计、综合性专业实验和毕业设计等实践环节让学 生边实践边自学, 培养学生的学习能力、实践能力和 理论联系实际的的综合能力。 


\section{4. 优选整合教育资源}

《教育法》鼓励：国家机关、企事业组织及其他 社会组织应当为学校组织的学生学习、社会实践活动 提供帮助和便利。这是充分利用社会资源提高人才培 养质量的一种有效途径, 而且, 教育资源的整合在一 定程度上能够缓解高校教育资源不足的困境。优选整 合教育资源的思路如图 4 所示。

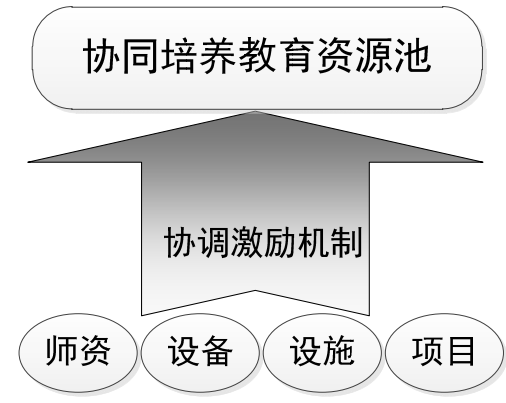

图 4 协同培养教育资源优选整合

优选整合教育资源实施主要有如下三种方法:

（1）归类优选。在科学技术高速发展的今天，高 校、企业均会投入一定技术和资金对新技术进行跟 踪、研发, 提升高校人才培养和企业产品价值的实效 性。以机器人为例, 很多企业或多或少会涉略该领域, 或研发制造, 或有效运用。因此, 有关行业最新技术 的教育资源（包括研发人员、设备、设施、项目）极 为丰富，而企业之间又多处于隔离封闭状态。所以， 对该类教育资源的整合必须进行归类优选，从运行体 量、维护能力、技术支持能力等多方面进行综合考量, 选取少部分优质教育资源进入协同培养资源池。

（2）跨界融合。基于互联网及新一代信息技术, 将分散在不同企业中的教育资源进行跨界整合, 由此 提供更大规模的有效教育资源。由于不同企业所处的 行业领域有所差异，因此这种跨界式的教育资源整 合, 为协同人才培养提供了综合性强、适应面广的工 程案例资源, 有利于培养工程人才的发散性思维, 创 造性地从不同工程领域的实践中抽取科学问题。

（3）师资交流。高校教师理论水平高、学术性强, 企业工程技术人员工程实践能力强, 两类人员作为最 重要的师资资源具有互补性。高校需要工程能力强的 师资介入, 以提高大学生的工程实践能力培养, 企业 需要学术性师资对长期积累的先进技术进行凝练提 升, 以增强企业的核心竞争力。通过师资的互动交流, 高校师资参与企业的工程项目, 企业师资参与高校教 学, 尤其是职业素养、实践实训类教学, 将形成真正 意义上的双赢局面。

在高校和行业协会推动下，教育资源的优选整合 是真正能够达到取长补短、互通有无的资源整合, 能 够为工科大学生培养提供宽视角、深度工程应用、创 业创新引导、职业道德和素养培养、工程能力深化和 提升等多方位的教育资源。

\section{5. 构建培养评估体系}

高等教育生命力和持续发展的关键是人才质量, 科学地评估人才培养质量能够充分反映人才培养体 系中存在的问题, 为给进一步教学改革方案制定提供 重要依据和引导方向。针对高校、协会、企业共同参 与的协同人才培养的特点, 结合行业专业技术人才职 业能力调查分析结果, 按照多主体所负责的职能, 制 订多方协同机制下的人才培养质量评估指标体系。

（1）评估对象。协同培养中的所有主体都必须纳 入到评估对象中, 包括学校、行业协会、企业和所培 养的人才。

（2）评估体系。从评估指标、评价依据和评分三 个维度进行细化, 不同主体的评估各有侧重点, 对学 校主要评估人才基础理论和素质培养效果、监督职责 完成情况、协同体系中工作完成情况等; 对协会主要 评估协调工作完成情况、对企业监督情况等; 对企业 主要评估课程完成质量情况、实践实训执行情况、监 督情况、人才职业素养培养情况等; 对所培养的人才 主要从双创能力、就业质量、用人单位反馈等多方面 进行评估。

（3）评估结果。为了提高评估的时效性, 可以引 入阶段性评价机制, 对执行效果不好的主体进行必要 的及时警示甚至淘汰。根据评估结果, 及时调整和解 决协同体系中人才培养计划、课程标准、校企课程内 容、工程能力培养环节等诸多方面存在的问题, 从而 不断优化工程技术人才培养体系。

\section{5. 结束语}

工科大学生的现代“科学-技术-工程”观培养之意 义可以归纳为以下三个方面:

（1）有助于学生树立正确的现代“科学-技术-工 程”观。增强工科大学生科学研究、技术创新与工程 实践的的自觉性，提高自身的科学素养、技术素养和 工程素养, 促使他们成为对经济社会发展有贡献的技 术型专业人才。

（2）有助于学校“新工科”建设和工程教育专业认 证。探讨“新工科”背景下大学生科技创新长效育人机 制, 推动学生的实践能力与创新能力培养, 构建“双 创”型人才培养模式和实践平台, 促进工科大学生的 就业和创业。

(3) 有助于探索新信息技术环境支撑下工程教育 方法。研究在物联网、移动互联网、云计划、大数据、 人工智能为代表的新一代信息技术支撑下，工程教育 教学的实施方式与有效方法, 尤其在特殊情况下, 使 学生切实掌握专业所需的 STE 知识与技能。

\section{致谢}

本文为佛山科学技术学院高等教育教学研究和改 革项目：工科大学生现代“科学-技术-工程”观的培养 
研究与实践、课程思政项目: 基于“敏感性”教育与培 养的《自动检测技术与自动化仪表》课程思政教学改 革研究的阶段性成果之一。

\section{REFERENCES}

[1] Yanjun Zhang, Zhenfeng Li. On STEM Problems in U.S. Higher Education and the Countermeasures in the 21st Century. Comparative Education Review. 2013(3):19-22.

[2] Committee on STEM Education, National Science and Technology Council. Federal Science, Technology, Engineering, and Mathematics (STEM) Education 5-Year Strategic Plan. http://www.whitehouse. gov/sites/default/files/microsites/ostp/stem_stratpla n_2013.pdf.

[3] Hui Luo, Zhaohui Li. Implementation Science, Technology, Engineering and Mathematics Education Strategic Plan to Enhance U.S. Competitiveness. Science Popularization, 2014(5):32-40.

[4] Zhongwen Cao. Knowing "Culinary Engineering” Based on the "Three Elements Theory of Science and Technology Engineering”. Journal of Researches on Dietetic Science and Culture. 2017(3):1-4.

[5] Shoudi Zhang. Science, Technology, Engineering and Higher Education. Journal of Changzhou Industrial Technology College (Natural Science Edition). 1998(4):79-83.

[6] CuiCui Tan. Exploration of Teaching Method Based on Network Teaching Platform. Science and Technology \& Innovation. 2020(16):71-72.

[7] Yi Liu. Design of Network Teaching Quality Monitoring System. Technology Innovation and Application. 2020(23): 84-85,88.

[8] Kelin Zhao. Reform Educational Plan and Improve the Quality of Engineering Science and Technology Talents. Shanghai Researches on Higher Education. 1986(1):33-37. 